Most of the accidents took place on highways (57.2\%), commonest being the road traffic accidents (55.1\%), brought by relatives (74\%). The mean duration for hospital stay was 5.42 days (SD \pm 8.312 days, range 1-79 days). $10 \%$ patients required resuscitation at the time of admission. Details of Glasgow coma scale were available; details regarding CT scan findings were available for 300 patients. Good recovery was seen in $68.4 \%$ and the mortality was in $7.2 \%$. Further details on vital parameters and investigations included in the study were also collected. Conclusions: TBI related research in many developing countries is in the developmental stages with relatively few published data. Although early analysis of a TBI data can lead to useful information, there is further need for the development of a user-friendly secure web-based database system to continuously maintain and analyze the registry.

Prehosp Disaster Med 2011;26(Suppl. 1):s19-s20

doi:10.1017/S1049023X1100077X

(A71) Prospective Evaluation of "Focused Assessment with Sonography for Trauma" Done by Emergency Physicians, and its Comparative Analysis with Radiologist's Performance

T.P. Sinha, ${ }^{1}$ S. Bhoi, ${ }^{2}$ S. Kumar, ${ }^{3}$ A. Bhasin,${ }^{4}$ M. Rodha ${ }^{1}$

1. Surgery, 110029, India

2. Emergency Medicine, New Delhi, India

3. Orthopedics, Delhi, India

4. Medicine, 110029 , India

Objective: The objective of this study was to determine the accuracy of emergency physicians in detecting free fluid in the abdomen when compared to radiologists during w primary survey of trauma victims by focused assessment with sonography for trauma (FAST) scan in the emergency department.

Methods: This prospective study was performed during a primary survey of the resuscitation of non-consecutive patients in the resuscitation bay. The study subjects included emergency physicians (EP) [one emergency medicine (EM) consultant, two EM residents, one orthopedic resident, and one surgical resident] who underwent training at a three-day workshop on emergency sonography and performed 10 supervised positive and negative scans for free fluid. The FAST scans were performed by the EPs and then by the radiology resident (RR). Both were blinded to each other's sonography findings. Computed tomography (CT) scan and laparatomy findings were used as gold standard. Results were compared between both groups. Intra-observer variability among EPs and level of agreement between EPs and RRs were assessed.

Results: One hundred fifty scans performed by EPs and RRs were analyzed. The mean age of the patients was 28 [1-70] years. Out of 24 true positive patients, 18 underwent CT scan, and exploratory laparatomy was done in six patients. Intra-observer performance variation ranged from $87-97 \%$. The sensitivity of FAST performed by EP and RR was $100 \%$. The specificity of FAST by EPs was $95.4 \%$ vs. $98.4 \%$ by RRs. The level of agreement was $100 \%$.

Conclusions: This study proves that FAST scan performed by EPs who are trained in short course of ultrasonography can be reliable and accurate when compared to a qualified radiologist. Prehosp Disaster Med 2011;26(Suppl. 1):s20

doi:10.1017/S1049023X11000781
(A72) Efficacy of Parental Opioid Analgesics versus NonOpioid Analgesic in Acute Pain Management of Trauma Victims in the Emergency Department G. Adhikari, ${ }^{1}$ S. Bhoi, ${ }^{2}$ P. Gautam, ${ }^{1}$ T.P. Sinha, ${ }^{1}$

M. Rodha, ${ }^{3}$ L. Kurrey, ${ }^{4}$ A. Bhasin ${ }^{3}$

1. Emergency Department, New Delhi, India

2. Department of Emergency Medicine, Trauma Centre, 110029, India

3. Surgery, Delhi, India

4. Emergency, Delhi, India

Background: The pyramid of pain management involves sequential drug escalation but its role is limited in an emergency department (ED). The efficacy of parental opioid analgesics versus non-opioid analgesic in acute pain management of trauma victims in the $\mathrm{ED}$ was evaluated to formulate protocol.

Methods: All alert patients with a baseline visual analogue scale score $(\geq 7)$ was randomly assigned either parental non-opioid (Group A) or opioid analgesics (Group B). The emergency care providers noted the VAS in either group at 15 minutes, 30 minutes, and 60 minutes, and at the time of discharge from the ED. If the patient's VAS score did not reduce by $50 \%$ at 30 minutes, repeat parental analgesics was given. The oral analgesics prescribed at the time of discharge were documented. Ethical clearance was taken. Data was compiled and analyzed.

Results: Of 106 patients, 99 were analyzed. The mean age in Group A was 33.2 \pm 13.2 years and $32.5 \pm 18$ years in Group B. The male:female ratio in Group A was 1.5:1 and 7:1 in Group B. The average baseline VAS score in Group A was 7.5, and that of Group B was 8.96. The average VAS at 15, 30, and 60 minutes and at discharge in Group A was 5.4, 5.34, 4.3, and 3.5 and it was 6.1, 6, 5.1, and 4.4. Repeat parental dose of analgesics were required in 95/99 (95\%) patients in Group A and 5\% that of Group B. The most common prescription at discharge from ED was non-opioid analgesics.

Conclusions: Acute pain relief was comparable in both groups. Non-opioid analgesics may be preferred over opioid in VAS score \pm 7 in a busy emergency department for early disposition.

Prehosp Disaster Med 2011;26(Suppl. 1):s20

doi:10.1017/S1049023X11000793

\section{(A73) Burden of Maxillofacial Trauma at Level Trauma 1} Centre

S. Sagar, M. Singhal, K. Kataria, S. Kumar, A. Gupta, B. Mishra Surgery, Delhi, India

Background: There is an upward trend in facial injuries following changes in population pattern, increasing industrialization and urbanization, hence maxillofacial trauma is becoming a burden and a leading medical problem in emergency rooms worldwide. Method: A retrospective study of patients with maxillofacial fractures seen and treated at the Jai Parkash Narayan Apex Trauma Center, AIIMS, New Delhi, India between January 2007 to June 2010. Data extracted from the patients' records include aetiology, age, sex, types and sites of fractures, treatment modality and concomitant injuries.

Results: There were 795 fractures of the maxillofacial skeleton and 86 concomitant injuries from 542 patients. Road traffic accident (56.8\%) was the most common aetiologic factor, followed by falls (22.3\%) and fights (18.5\%). The age range was from 3 years to 75 years (mean $=34.7)$ with a peak incidence in the $3^{\text {rd }}$ decade 